\title{
Utilizando Splines Cúbicas Naturais para Atenuação de Erros no Posicionamento GPS ${ }^{1}$
}

\author{
D.B.M. ALVES ${ }^{2}$, M. MENEGUETTE Jr. ${ }^{3}$,J.F.G. MONICO ${ }^{4}$, FCT-UNESP, Campus \\ de Presidente Prudente, 19060-900 Presidente Prudente, SP, Brasil.
}

\begin{abstract}
Resumo. O GPS (Global Positioning System) é um sistema de posicionamento global e de radionavegação que tem como principal objetivo viabilizar o posicionamento de baixa, média e alta precisão. Mas as observáveis GPS estão sujeitas a erros que degradam o posicionamento. Para atenuar os efeitos desses erros trabalhos recentes têm utilizado o modelo semiparamétrico e o método dos mínimos quadrados (MMQ) com penalidades. No modelo semiparamétrico as variáveis estimadas são divididas em uma parte paramétrica, que é de interesse do usuário, e uma parte não-paramétrica (funções de erros que variam suavemente com o tempo). Para tal modelo, utiliza-se o MMQ com penalidades. Essa técnica utiliza uma spline cúbica natural, cuja suavidade é determinada pelo parâmetro suavizador, calculado pela validação cruzada generalizada. Nesse método, os erros são modelados como funções que variam suavemente com o tempo. E mais, as funções de erros sistemáticos, ambigüidades e coordenadas de interesse são estimadas simultaneamente. Como resultado, as ambigüidades e as coordenadas de interesse são estimadas com melhor confiança e acurácia do que com o MMQ convencional.
\end{abstract}

\section{Introdução}

O GPS é um sistema de abrangência global que permite ao usuário, em qualquer local da superfície terrestre, ou próximo a ela, dispor de no mínimo quatro satélites para serem rastreados, sem necessidade de intervisibilidade entre as estações, e independente das condições climáticas. Esse sistema tem como principal objetivo viabilizar a navegação de baixa, média e alta precisão [13]. Além disso, o GPS tem se tornado uma tecnologia extremamente útil e inovadora para uma série de atividades que necessitam de posicionamento.

No entanto, as observáveis GPS, que permitem determinar posição, velocidade e tempo, tal como todas as outras observáveis envolvidas nos processos de medidas, estão sujeitas a erros aleatórios, sistemáticos e grosseiros. Para obter resultados confiáveis, o modelo matemático estabelecido deve ser válido para a realidade física

\footnotetext{
${ }^{1} \mathrm{~A}$ primeira autora tem bolsa de estudos fornecida pela FAPESP.

${ }^{2}$ danibarroca@yahoo.com.br, aluna de Doutorado do Programa de Pós Graduação em Ciências Cartográficas.

${ }^{3}$ messias@prudente.unesp.br, Departamento de Matemática, Estatística e Computação.

${ }^{4}$ galera@prudente.unesp.br, Departamento de Cartografia.
} 
que se tenta descrever e ser capaz de detectar problemas nas observáveis utilizadas. Dessa forma, todos os tipos de erros envolvidos no processo de medida devem ser considerados.

Os erros aleatórios são inevitáveis, sendo, portanto, considerados uma propriedade inerente das observações [7]. Erros grosseiros (outliers) devem ser eliminados através do processo de controle de qualidade [17]. Erros sistemáticos podem ser parametrizados, isto é, modelados como parâmetros adicionais, ou eliminados por técnicas apropriadas de observação e/ou processamento. Erros sistemáticos podem degradar a acurácia dos resultados [1].

Para atenuar os efeitos dos erros sistemáticos e para melhorar a confiabilidade da estimativa das coordenadas de interesse, estudos têm sido realizados no sentido de se aplicar o método dos Mínimos Quadrados com Penalidades (MMQ com penalidades) [11], dentro do contexto do modelo semiparamétrico, usando uma spline cúbica natural [9].

Nesse artigo, o MMQ com Penalidades e o modelo semiparamétrico são utilizados para atenuar erros sistemáticos no posicionamento GPS. Além disso, é realizada uma revisão teórica dos principais aspectos envolvidos nesse tipo de processamento.

\section{Erros Sistemáticos}

Erros sistemáticos degradam a confiabilidade e acurácia do posicionamento realizado com o GPS. Dentre esses erros as principais fontes são a refração ionosférica e troposférica, os erros nas órbitas dos satélites GPS e o multicaminho.

A ionosfera é um meio dispersivo para a faixa de freqüência do GPS, no qual o índice de refratividade é função da freqüência utilizada. Além disso, a ionosfera apresenta variações diárias, sazonais, geográficas e de longo período [6], o que dificulta sua modelagem. A troposfera é um meio não dispersivo. Usualmente, as componentes úmida e hidrostática (seca) expressam a influência da troposfera nas medidas GPS [16]. Os erros da ionosfera podem variar de poucos metros a dezenas de metros, enquanto que na troposfera os erros no zênite estão geralmente entre dois e três metros [12].

Informações sobre as órbitas dos satélites GPS podem ser obtidas através das efemérides transmitidas pelos satélites ou das efemérides precisas fornecidas pelo International GPS Service (IGS). A partir das efemérides são calculadas as posições dos satélites GPS, normalmente, injuncionadas como fixas durante o processo de ajustamento dos dados GPS. Assim, erros nas coordenadas dos satélites se propagam para a posição do usuário.

O multicaminho é o fenômeno pelo qual um sinal chega à antena do receptor por caminhos múltiplos, devido à reflexão. É causado, principalmente, por reflexões do sinal em superfícies próximas ao receptor, tais como construções, carros, árvores, colinas, etc [13]. Efeitos secundários são causados por reflexões no próprio satélite e durante a propagação do sinal [10]. A magnitude dos erros depende da geometria do cenário que envolve as antenas, os satélites e os objetos refletores e da natureza do material reflexivo [4]. O erro causado pelo multicaminho pode degradar a acurácia das coordenadas em alguns centímetros [15]. 


\section{Splines Cúbicas Naturais}

Para se trabalhar com o ajustamento e interpolação de funções, a aproximação por polinômios é muito conveniente, uma vez que os polinômios têm várias propriedades interessantes, dentre estas a de função analítica, que torna possível calcular as derivadas, de qualquer ordem, dos polinômios. Entretanto, a necessidade de muitas derivadas da função que está sendo aproximada por polinômios pode ser muito restritiva [3].

Uma solução para isso é a utilização de polinômios por partes, pois dessa forma são permitidas descontinuidades das derivadas de ordem mais elevadas em alguns pontos. Essa característica "pseudo analítica" confere às funções polinomiais por partes, chamadas splines, boas propriedades de aproximação, convergência e estabilidade.

Matematicamente, dados os números reais $t_{1}, \ldots, t_{n}$ em algum intervalo $[a, b]$, satisfazendo $a<t_{1}<t_{2}<\ldots<t_{n}<b$, uma função $g$ definida sobre $[a, b]$ é uma spline cúbica se em cada intervalo $\left(a, t_{1}\right),\left(t_{1}, t_{2}\right), \ldots,\left(t_{n}, b\right), g$ é um polinômio cúbico e a primeira e segunda derivadas são contínuas em cada $t_{i}$ e, portanto, contínuas no intervalo $[a, b]$. Uma spline cúbica sobre um intervalo $[a, b]$ será dita uma spline cúbica natural se as segunda e terceira derivadas são nulas em $a$ e $b$ [1].

A spline cúbica natural é utilizada no MMQ com Penalidades. Para tanto, duas matrizes, $Q$ e $R$, advindas da definição de spline cúbica natural, serão definidas a seguir.

Seja $h_{i}=t_{i+1}-t_{i}$ para $i=1, \ldots, n-1$. Seja $Q$ uma matriz $n \times(n-2)$ com entradas $q_{i j}$, para $i=1, \ldots, n$ dada por [1]:

$$
q_{j-1, j}=h_{j-1}^{-1}, \quad q_{j j}=-h_{j-1}^{-1} \quad \text { e } q_{j+1, j}=h_{j}^{-1},
$$

para $j=2, \ldots, n-1$ e $q_{i j}=0$ para $|i-j| \geq 2$. A matriz simétrica $R$ é $(n-2) \times(n-2)$ com elementos $r_{i j}$ dados por:

$$
\begin{aligned}
r_{i i} & =\frac{1}{3}\left(h_{i-1}+h_{i}\right), \quad i=2, \ldots, n-1, \\
r_{i, i+1} & =r_{i+1, i}=\frac{1}{6} h_{i}, \quad i=2, \ldots, n-2
\end{aligned}
$$

e $r_{i j}=0$ para $|i-j| \geq 2$.

A matriz $R$ é estritamente diagonal dominante, pois $\left|r_{i i}\right|>\sum_{j \neq i}\left|r_{i j}\right|$ para cada $i$. Assim, usando o teorema de Gershgorin [8], todos os autovalores de $R$ são positivos. Portanto, $R$ é estritamente positiva definida, e conseqüentemente, é inversível. Pode-se então definir uma matriz $K$ dada por:

$$
K=Q R^{-1} Q^{T}
$$

Utilizando a matriz $K$ pode-se definir o termo de penalidade de aspereza que é utilizado no MMQ com Penalidades,

$$
\int_{a}^{b} g "(t)^{2} d t=g^{T} K g,
$$

onde $g$ é o vetor $\left(g_{1}, \ldots, g_{n}\right)^{T}$. 


\section{Modelo Semiparamétrico e Método dos Mínimos Quadrados com Penalidades}

No modelo semiparamétrico, o cálculo das variáveis é dividido em duas partes: a parte paramétrica e a não-paramétrica. Normalmente, a parte paramétrica é a de interesse do usuário. Já a parte não-paramétrica representa uma combinação de algumas funções de erros que variam suavemente com o tempo [1].

$\mathrm{O}$ vetor semiparamétrico pode ser expresso por [2]:

$$
y_{i}=A_{i} x+M_{i} g\left(t_{i}\right)+\varepsilon_{i} \quad i=1,2, \ldots, n .
$$

Na equação (4.1): $y_{i}$ são as observações na i-ésima época; $A_{i}$ é a matriz dos coeficientes da equação (matriz design); $x$ é o vetor dos parâmetros a ser estimado; $M_{i}$ é a matriz de incidência; $g\left(t_{i}\right)$ são as funções de erros; $t_{i}$ é o índice de tempo; $\varepsilon_{i}$ é o vetor dos erros aleatórios na i-ésima época; $n$ é o número de épocas.

Dois casos podem ser considerados para a equação (4.1) . Primeiro, se o número de incógnitas é maior que o número de observações. Neste caso, a equação (4.1) não pode ser resolvida usando o MMQ convencional. Já no outro caso, mesmo com o número de incógnitas menor que o número de observações, a equação (4.1) pode não fornecer uma solução estável quando o MMQ convencional é usado [11].

Para que se obtenha uma solução confiável, injunções adicionais, que correspondem à penalidade do ajustamento, devem ser adicionadas (equação (3.1)). Trata-se do MMQ com penalidades. Dessa forma, a função a ser minimizada é dada por [11]:

$$
\begin{aligned}
& \sum_{i=1}^{n}\left\{\left(y_{i}-A_{i} x-M_{i} g\left(t_{i}\right)\right)^{T} P_{i}^{-1}\left(y_{i}-A_{i} x-M_{i} g\left(t_{i}\right)\right)\right\}+\sum_{j=1}^{q} \alpha_{j} \int\left(g_{j}^{\prime \prime}(t)\right)^{2} d t \\
= & \min ,
\end{aligned}
$$

onde $P_{i}^{-1}$ é a matriz peso das observações, $\alpha_{j}$ é o parâmetro suavizador, $g_{j}^{\prime \prime}(t)$ é a segunda derivada da j-ésima função em relação ao tempo e $q$ é o número de funções de erros.

A equação (4.2) define a forma quadrática penalizada. A primeira parte da equação (4.2) refere-se a forma quadrática dos resíduos do MMQ, e a segunda parte ao termo de penalidade de aspereza. Mas, para desenvolver os cálculos computacionalmente, a penalidade de aspereza pode ser expressa por [5]:

$$
\begin{aligned}
& \sum_{j=1}^{q} \alpha_{j} \int\left(g{ }_{j}(t)\right)^{2} d t \\
= & \left(\left(R^{-1} \otimes I_{q}\right)\left(Q^{T} \otimes I_{q}\right) g\right)^{T}(R \otimes D(\alpha))\left(\left(R^{-1} \otimes I_{q}\right)\left(Q^{T} \otimes I_{q}\right)\right) g,
\end{aligned}
$$

onde $Q$ e $R$ são matrizes relacionadas com o índice de tempo $t_{i}$ (seção 3 ); $I_{q}$ é a matriz identidade $q \times q ; D(\alpha)=\operatorname{diag}\left(\alpha_{1}, \ldots, \alpha_{q}\right) ; \otimes$ denota o produto de Kronecker.

Dessa forma, substituindo (4.3) em (4.2), tem-se:

$$
\sum_{i=1}^{n}\left\{\left(y_{i}-A_{i} x-M_{i} g\left(t_{i}\right)\right)^{T} P_{i}^{-1}\left(y_{i}-A_{i} x-M_{i} g\left(t_{i}\right)\right)\right\}
$$




$$
\begin{aligned}
& +\left(\left(R^{-1} \otimes I_{q}\right)\left(Q^{T} \otimes I_{q}\right) g\right)^{T}(R \otimes D(\alpha))\left(\left(R^{-1} \otimes I_{q}\right)\left(Q^{T} \otimes I_{q}\right)\right) g \\
& =\min
\end{aligned}
$$

Minimizando em relação a $x$ e $g$, obtém-se respectivamente:

$$
\begin{aligned}
& A^{T}\left(I_{n} \otimes P^{-1}\right) A x+A^{T}\left(I_{n} \otimes P^{-1}\right)\left(I_{n} \otimes M\right) g=A^{T}\left(I_{n} \otimes P^{-1}\right) y, \\
& \quad\left(I_{n} \otimes M^{T}\right)\left(I_{n} \otimes P^{-1}\right) A x+\left(\left(I_{n} \otimes M^{T}\right)\left(I_{n} \otimes P^{-1}\right)\left(I_{n} \otimes M\right)\right. \\
& \left.\quad+(Q \otimes D(\alpha))\left(R^{-1} \otimes I_{q}\right)\left(Q^{T} \otimes I_{q}\right)\right) g \\
& \quad=\left(I_{n} \otimes M^{T}\right)\left(I_{n} \otimes P^{-1}\right) y,
\end{aligned}
$$

sendo $A=\left(A_{1}, A_{2}, \ldots, A_{n}\right)^{T} ; P^{-1}=P_{1}^{-1}=\ldots=P_{n}^{-1}$, considerando a mesma constelação GPS; $g=\left(g_{1}^{1}, \ldots g_{1}^{q}, \ldots, g_{n}^{1}, \ldots, g_{n}^{q}\right)$.

As equações (4.4) e (4.5) podem ser resolvidas pelo método direto [9], ou seja, substituindo a equação (4.5) na equação (4.4). Assim, utilizando o método direto em tais equações e realizando algumas manipulações matemáticas, tem-se:

$$
x=\left[A^{T}\left(I_{n} \otimes P^{-1}\right) A-A^{T}\left(I_{n} \otimes P^{-1}\right) A_{S}\right]^{-1}\left[A^{T}\left(I_{n} \otimes P^{-1}\right) y-A^{T}\left(I_{n} \otimes P^{-1}\right) y_{S}\right],
$$

onde $A_{S}=S A$ é a matriz design suavizada; $y_{S}=S y$ é o vetor de observações suavizadas; $S$ é a matriz de suavização dada por:

$S=(I \otimes M)\left(\left(I \otimes M^{T}\right)\left(I_{n} \otimes P^{-1}\right)+(Q \otimes D(\alpha))\left(R^{-1} \otimes I_{q}\right)\left(Q^{T} \otimes I_{q}\right)\right)^{-1}\left(I \otimes M^{T}\right)\left(I_{n} \otimes P^{-1}\right)$.

\section{Parâmetro Suavizador}

Existem duas filosofias diferentes para a escolha do parâmetro suavizador. No primeiro procedimento, considera-se a escolha livre do parâmetro suavizador $(\alpha)$ um critério vantajoso. Variando o parâmetro suavizador, algumas características dos dados podem ser exploradas. Mas, se uma única estimativa é desejada, esta pode ser obtida através de uma escolha subjetiva. Já o outro procedimento se opõe ao primeiro pois, nesse caso, a escolha do parâmetro suavizador deve ser automática, isto é, calculado a partir dos dados [1].

O procedimento automático é essencial em aplicações onde o método é aplicado para um grande número de conjuntos de dados, ou quando esse método é um componente de um processo mais complicado. Portanto, nesse trabalho um método automático é utilizado para escolher o parâmetro suavizador: a validação cruzada generalizada (Generalized Cross Validation - GCV). A Figura 1 ilustra um caso onde o parâmetro suavizador é escolhido pela GCV. Note que a spline acompanha a tendência dos dados. Pode-se acrescentar que se $\alpha$, escolhido arbitrariamente, é pequeno, a curva se aproximará de uma interpolação (Figura 2). Caso contrário, se for muito grande, a curva se aproximará de uma regressão linear (Figura 3). Para 
mais detalhes consulte [1].

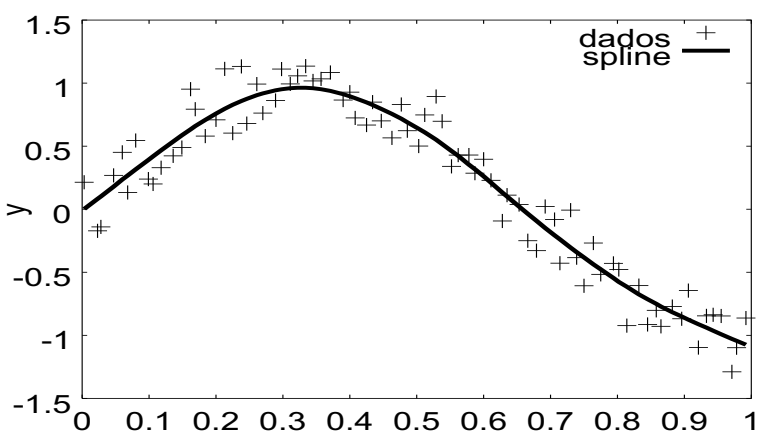

Figura 1: $\alpha$ escolhido pela $\mathrm{GCV}$

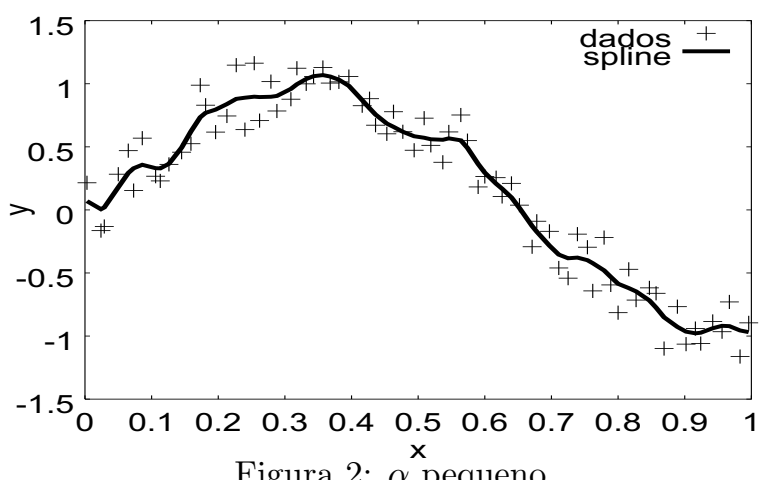

Figura 2: $\alpha$ pequeno

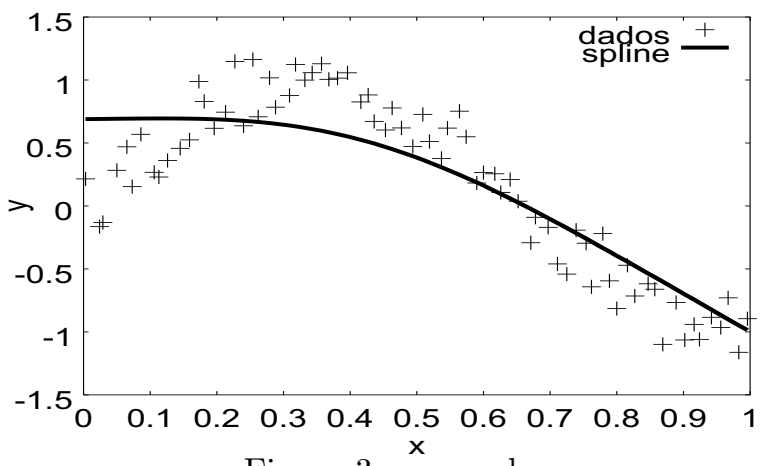

Figura 3: $\alpha$ grande

A função de validação cruzada generalizada é dada por [9]:

$$
G C V(\alpha)=\frac{1}{n} \frac{\left(y-A x-\left(I_{n} \otimes M\right) g\right)^{T}\left(I_{n} \otimes P^{-1}\right)\left(y-A x-\left(I_{n} \otimes M\right) g\right)}{(m-\operatorname{tr} H / n)^{2}}
$$


onde $n$ é o número de épocas; $m$ é o número de observações por época; $H$ é a matriz dada por:

$$
H(\alpha)=S+\left(I_{n m}-S\right) A\left(A^{T}\left(I_{n} \otimes P^{-1}\right)\left(I_{n m}-S\right) A\right)^{-1} A^{T}\left(I_{n} \otimes P^{-1}\right)\left(I_{n m}-S\right) .
$$

A escolha do parâmetro suavizador na GCV é realizada pela minimização da função GCV em $\alpha$. Por isso, um método de minimização deve ser utilizado. Nesse trabalho foi utilizado o método Gold Search [14].

\section{Experimento Realizado e Análises}

Para testar o MMQ com Penalidade e o modelo semiparamétrico, dados da linha de base UEPP-ASSIS $(\sim 116 \mathrm{~km})$ foram utilizados. UEPP é uma estação permanente da Rede Brasileira de Monitoramento Contínuo (RBMC), localizada no interior do estado de São Paulo, cujas coordenadas são conhecidas. A estação ASSIS não possui coordenadas conhecidas, o objetivo nesse trabalho é determiná-las.

É necessário ressaltar que pesquisadores da FCT/UNESP estão desenvolvendo um software para processamento de dados GPS, chamado GPSeq. O GPSeq foi implementado com o MMQ convencional recursivo (MMQ). No entanto, o MMQ com Penalidades (MMQP), desenvolvido pela autora, também foi implementado no GPSeq. Portanto, nesse artigo, os resultados obtidos pelo MMQ e o MMQP foram comparados.

Quatro conjuntos de dados, dois com 5 min e dois com 10 min de ratreio, foram processados. Os dados utilizados foram coletados por volta das $14 \mathrm{~h}$ local, momento no qual os efeitos causados pela ionosfera são mais relevantes. Além disso, 7 satélites foram rastreados. As Figuras 4 e 5 ilustram as discrepâncias em relação às coordenadas, $\mathrm{E}$ (leste), $\mathrm{N}$ (norte) e h (altura), consideradas verdadeiras para 5 min e 10 min respectivamente. As coordenadas consideradas verdadeiras foram calculadas em um software científico utilizando $6 \mathrm{~h}$ de dados coletados durante a noite.

Note que para todos os conjuntos de dados, as discrepâncias obtidas pelo modelo semiparamétrico utilizando o MMQP em relação às coordenadas verdadeiras são muito menores do que no MMQ, mostrando a eficiência do método proposto em um curto intervalo de tempo de processamento dos dados.

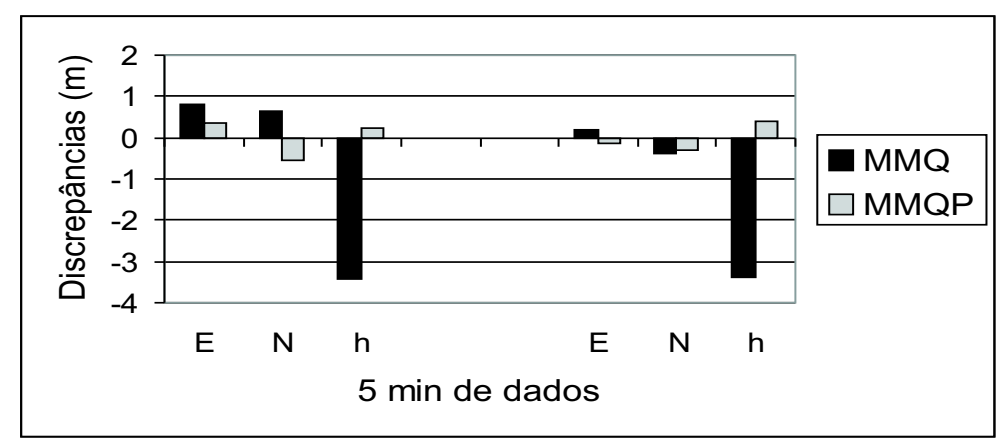

Figura 4: Discrepâncias para 5 min de dados 


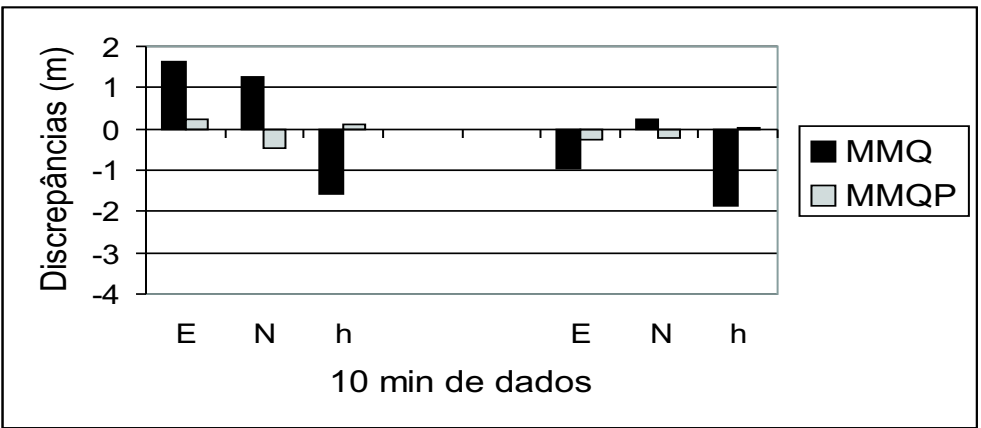

Figura 5: Discrepâncias para 10 min de dados

\title{
7. Conclusão
}

Neste artigo, o MMQ com Penalidades e o modelo semiparamétrico foram utilizados para atenuar erros sistemáticos no posicionamento relativo GPS. Para tanto, uma revisão teórica foi realizada com o objetivo de contemplar os principais tópicos envolvidos nessa técnica, em especial, as splines cúbicas naturais e a escolha do parâmetro suavizador.

Os resultados mostraram que em todos os intervalos de dados, as discrepâncias das coordenadas obtidas pelo MMQ com Penalidades foram menores se comparadas com o MMQ convencional. Com isso, pode-se concluir que o método proposto é eficiente na mitigação de erros sistemáticos no posicionamento relativo GPS.

\begin{abstract}
The GPS (Global Positioning System) is a global positioning and radio navigation system that has as main goal to make available the low, medium and high precision positioning. But, the GPS observables are subject to errors that degrade the positioning. Mitigating these errors some recent works have been using the semiparametric model and the penalised least squares technique. In a semiparametric model the estimated variables are divided into a parametric part, which is of interest to the users, and a nonparametric one (composed by error functions that vary smoothly with time). So, the penalised least squares is used. This technique uses a natural cubic spline, whose smoothness is determined by a smoothing parameter, computed by using the generalized cross validation. In this method, the errors are modeled as functions which vary smoothly in time. And more, the systematic errors functions, ambiguities and station coordinates are estimated simultaneously. As a result, the ambiguities and the station coordinates are estimated with better reliability and accuracy than the conventional least square method
\end{abstract}

\section{Referências}

[1] D.B.M. Alves, "Método dos Mínimos Quadrados com Penalidades: Aplicação no Posicionamento Relativo GPS", Dissertação de Mestrado, UNESP, Presidente Prudente, SP, 2004. 
[2] D.B.M. Alves, Using cubic splines to mitigate systematic errors in GPS relative positioning, em "ION GNSS 2004", Long Beach, Califórnia, 2004.

[3] C.M. Cunha, "Métodos Numéricos", 2.ed. Unicamp, 2000.

[4] J.C. Farret, "O Efeito do Multicaminho Estático nas Medidas da Fase das Portadoras GPS", Tese de Doutorado, Universidade Federal do Paraná, Curitiba, 2000 .

[5] J.A. Fessler, Nonparametric fixed-interval smoothing with vector splines, em "IEEE Transactions on Signal Processing", Proceedings Vol.39, pp. 852-859, 1991.

[6] L.P.S. Fortes, "Optimising the Use of GPS Multi-Reference Stations for Kinematic Positioning", Tese de Doutorado, University of Calgary, Calgary, 2002.

[7] C. Gemael, "Introdução ao Ajustamento de Observações: Aplicações Geodésicas", 1994, 319p.

[8] G.H. Golub e C.F.V. Loan, "Matrix Computations", Johns Hopkins University Press, 1983.

[9] P.J. Green e B.W. Silverman, "Nonparametric Regression and Generalized Linear Models: a Roughness Penalty Approach", 1. ed., Chapman \& Hall, London, 1994.

[10] B.H. Wellenhof, H. Lichtenegger e J. Collins, "GPS Theory and Practice", 4. ed., Springer-Verlag, Wien, 1997.

[11] M. Jia, M. Stewart e M. Tsakiri, Mitigation of ionospheric errors by penalised least squares technique for high precision medium distance GPS positioning, em "KIS 2001", Canada, 2001.

[12] J.A. Klobuchar, Ionospheric Effects on GPS, em "Global Positioning System: Theory and Applications" (B.W. Parkinson e J.J. Spilker Jr., eds.), v.1, pp. 485-515, 1996.

[13] J.F.G. Monico, "Posicionamento pelo NAVSTAR-GPS: Descrição, Fundamentos e Aplicações", 1. ed., Unesp, São Paulo, 2000.

[14] W.H. Press et al., "Numerical Recipes: The Art of Scientific Computing", Cambridge University Press, New York, 1986.

[15] J.K. Ray, "Mitigation of GPS Code and Carrier Phase Multipath Effects Using a Multi-Antenna System", Dissertação de Mestrado, University of Calgary, Calgary, 2000.

[16] L.F. Sapucci, "Estimativa do Vapor d'água Atmosférico e a Avaliação do Atraso Zenital Troposférico Utilizando GPS", Dissertação de Mestrado, UNESP, Presidente Prudente, SP, 2001.

[17] P.J.G. Teunissen e A. Kleusberg, "GPS for Geodesy", 2ed., Springer-Verlag, Berlin, 1998. 
